\title{
Handgrip Endurance in Healthy Elderly People
}

\author{
So Miyahara*, Yoshihiro Tanikawa, Hideo Hirai, Seiji Togashi \\ Department of Rehabilitation, Faculty of Health Sciences, Hiroshima Cosmopolitan University, Hiroshima, Japan.
}

*Corresponding author: So Miyahara, Department of Rehabilitation, Faculty of Health Sciences, Hiroshima Cosmopolitan University, 3-2-1 Othukahigashi, Asaminami ward, Hiroshima, 731-3166, Japan.
Received Date: May 22, 2020

Published Date: June 4, 2020

\begin{abstract}
In general, there are many items for measuring physical function. Among them, handgrip strength is easy to use because it can be easily measured in a short time ( 3 to 5 seconds). There are few reports that evaluate handgrip endurance, we reported it.

The subjects were 16 people $(78.2 \pm 3.7$ years old) living in Hiroshima City. Using a grip strength meter T.K.K.5401 (Takei Kiki Co., Ltd.), the maximum force in the dominant handgrip strength was measured while holding for 20 seconds, and the average value per second was calculated from the obtained results. As a comparison group, 11 students (21.5 \pm 0.5 years old, hereafter: young group) enrolled also performed similar measurements.
\end{abstract}

Result: The maximum grip strength was significantly lower in the elderly group than in the young group. The change in grip strength for 20 seconds in each group was significantly lower than the maximum value in the young group after 16 seconds had passed since the start of the grip exercise. On the other hand, in the elderly group, no decrease in grip strength was observed within 20 seconds after the start of the grip exercise (interaction effect $\mathrm{p}<0.01$ ). Comparing the transition of the elapsed time from the maximum value, the young group showed a significant decrease after 12 seconds from the start, but the elderly group did not show a significant decrease.

Conclusion: The elderly group did not show a significant decrease in handgrip endurance for 20 seconds. When momentary exertion of power is required in daily life, if the purpose cannot be achieved, other means and ingenuity are required to achieve the purpose. In the future, I would like to find out the relevance and risk with evaluation other items, whether it will be one index in physical fitness tests.

Keywords: Handgrip Endurance;Elderly

Abbreviations ED: Handgrip Endurance

\section{Introduction}

There are many evaluation methods that can measure physical function for many peoples [1-4]. The assessment of sarcopenia and flail is of great clinical and research interest and is growing rapidly[5,6].The theory to be related to these two evaluations has also been clarified, and in recent years, a new standard for sarcopenia has been created, and it is also used in hospitals and exercise facilities used by the elderly[6]. Among them, the hand grip strength is particularly easy to use because it can be easily measured in a short time.The Asian Working Group for Sarcopenia (AWGS)

explains that the standard value of grip strength of sarcopenia, which is considered to be low muscle strength, is less than $28 \mathrm{~kg}$ for men and less than $18 \mathrm{~kg}$ for women[6]. Lifestyle interventions in the elderly, especially exercise and nutrition, have become the mainstay of treatment to improve this low muscle strength. These operations are also preventive measures for the elderly requiring long-term care, which are faced by countries entering a superaging society.There are many evaluation items based on grip strength[7-9], but few studies have evaluated handgrip endurance 
(ED) [10]. In addition, there are no studies that measured and compared the difference in ED changes between the elderly and young people, and the change with elapsed time (seconds). And there are few reports that examined the measurement time of grip strength in the elderly.

Therefore, the purpose of this study was to compare and consider the changes in ED of healthy elderly people and ED of young people with elapsed time (seconds) and changes in grip strength measurement time, respectively.

\section{Materials and Methods}

The subjects were 16 elderly people aged 65 and over ( 2 males, 14 females, $78 \pm 3.8$ years old) who performed light intensity exercises once a week. They all live in Hiroshima City, Hiroshima Prefecture. As a comparison group, there were 11 healthy young people ( 5 males, 6 females, $21.5 \pm 0.5$ years old). The measurement was performed in January 2020, paying attention to the prevention of the spread of infectious diseases caused by COVID-19. Subjects with diseases that may affect grip strength measurement were excluded (patients with severe heart failure, end-stage renal disease, malignant tumors, dementia, severe chronic lung disease, and those unable to walk $20 \mathrm{~m}$ or more). This study was anonymized based on the protection of personal information and data was obtained with the approval by Hiroshima Cosmopolitan University Ethics Committee (2019004).

For grip strength measurement, a digital grip strength meter (T.K.K.5401 Takei Kikai Kogyo) was digitally output using a strain amplifier (T.K.K.1268 Takei Kikai Kogyo) and an A/D converter (T.K.K.5721 Takei Kikai Kogyo) for measurement and analysis.

The sampling rate of Handgrip Endurance was set to $1 / 100$, and the average value of the measured results was calculated per second. The subjects did not have a backrest in the chair, bent their knees at right angles[11], placed their soles on the ground, and bent their elbows on the measuring side at right angles, and measured their dominant hand with maximum force for 20 seconds. Then, the maximum grip strength value and the average value per second were calculated. The subjects measured calf circumference in the same posture[11].

Commercially available software (SPSS ver.26 IBM) was used for the analysis. In the data, the table shows the mean \pm SD and the figure shows the mean \pm SE. Continuous variables are displayed as average values. $p$ value of less than 0.05 was considered statistically significant.

\section{Result and Discussion}

\section{Result}

There were significant differences in social background (age and BMI) and maximum grip strength between the elderly and young groups $(21.3 \pm 5.3 \mathrm{~kg}$ vs. $38.9 \pm 11.1 \mathrm{~kg}, \mathrm{p}<0.05)$. The continuous grip strength for 20 seconds was significantly different between the elderly group and the young group (Interaction effect $\mathrm{p}<0.01$ ). The average grip strength value per second was the maximum after 3 seconds for both the elderly and the young. The younger ones showed a significant decrease from the maximum value after 16 seconds, but the elderly did not. The rate of decrease per second with respect to the maximum value was also significantly different between the elderly group and the young group (interaction effect $\mathrm{p}<0.01$ ). In the young group, the rate of decrease was small 3 seconds after the start and decreased significantly after 12 seconds. In the elderly group, the rate of decrease was small 3 seconds after the start and decreased significantly after 16 seconds.(Figure 1,2) (Table 1).

Table 1: People Characteristics, Physical Function Data

\begin{tabular}{|c|c|c|c|c|}
\hline & All & Elderly Group & Younger Group & P Value \\
\hline Number & 27 & 16 & 11 & \\
\hline Male gender,n(\%) & $7(25.9)$ & $2(12.5)$ & $5(45.5)$ & 0.06 \\
\hline Age, yrs & $77.5 \pm 3.5$ & $78.2 \pm 3.7$ & $21.5 \pm 0.5$ & $<0.01$ \\
\hline Body mass index, $\mathrm{kg} / \mathrm{m} 2$ & $22.4 \pm 2.8$ & $23.1 \pm 3.4$ & $21.3 \pm 1.0$ & 0.06 \\
\hline \multicolumn{5}{|c|}{ Social Background, N(\%) } \\
\hline Car user & $9(33.3)$ & $3(42.9)$ & $3(34.6)$ & 0.6 \\
\hline Housework & $12(44.4)$ & $12(75.0)$ & $0(0)$ & $<0.01$ \\
\hline fall down history within 1 years & $1(3.7)$ & $1(6.3)$ & $0(0)$ & 0.42 \\
\hline \multicolumn{5}{|c|}{ Comorbidities, n(\%) } \\
\hline Hypertension & $5(18.5)$ & $5(31.3)$ & $0(0)$ & 0.04 \\
\hline Dyslipidemia & $7(25.9)$ & $7(43.8)$ & $0(0)$ & $<0.01$ \\
\hline \multicolumn{5}{|c|}{ Medication, $\mathrm{n}(\%)$} \\
\hline ARB/ACE-inhibitors & $7(53.8)$ & $5(31.3)$ & $0(0)$ & 0.59 \\
\hline
\end{tabular}




\begin{tabular}{|c|l|c|c|c|}
\hline & $2(15.4)$ & $3(14.3)$ & $0(0)$ & 0.9 \\
\hline CCB & $7(25.9)$ & $7(43.8)$ & $0(0)$ & 0.85 \\
\hline Statin & Physical function calf circumference, cm & $34.9 \pm 2.8$ & 0.01 \\
\hline \multicolumn{7}{|c|}{ Handgrip strength, kg } \\
\hline Rt & $34.9 \pm 2.8$ & $33.9 \pm 2.9$ & $36.4 \pm 1.9$ & 0.01 \\
\hline Lt & $34.9 \pm 2.6$ & $21.5 \pm 5.3$ & $38.9 \pm 11.1$ & $<0.01$ \\
\hline Rt & $28.0 \pm 11.7$ & $20.1 \pm 5.0$ & $35.8 \pm 11.2$ & $<0.01$ \\
\hline Lt & $23.1 \pm 11.0$ & &
\end{tabular}

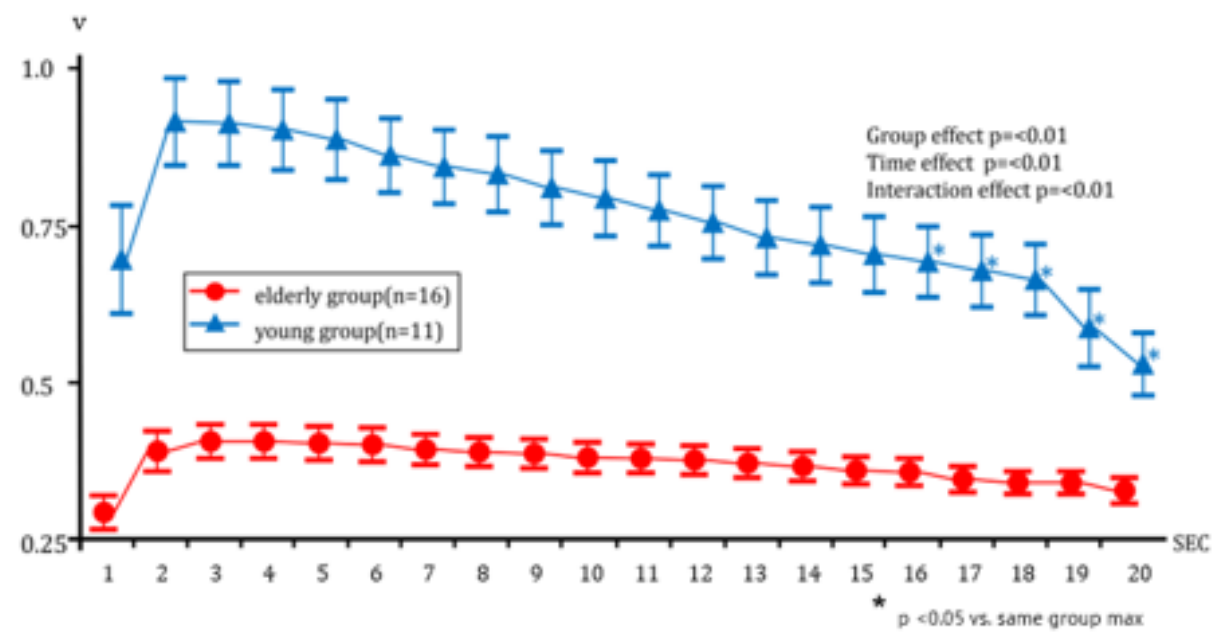

Figure 1: Changes in handgrip strength per second.

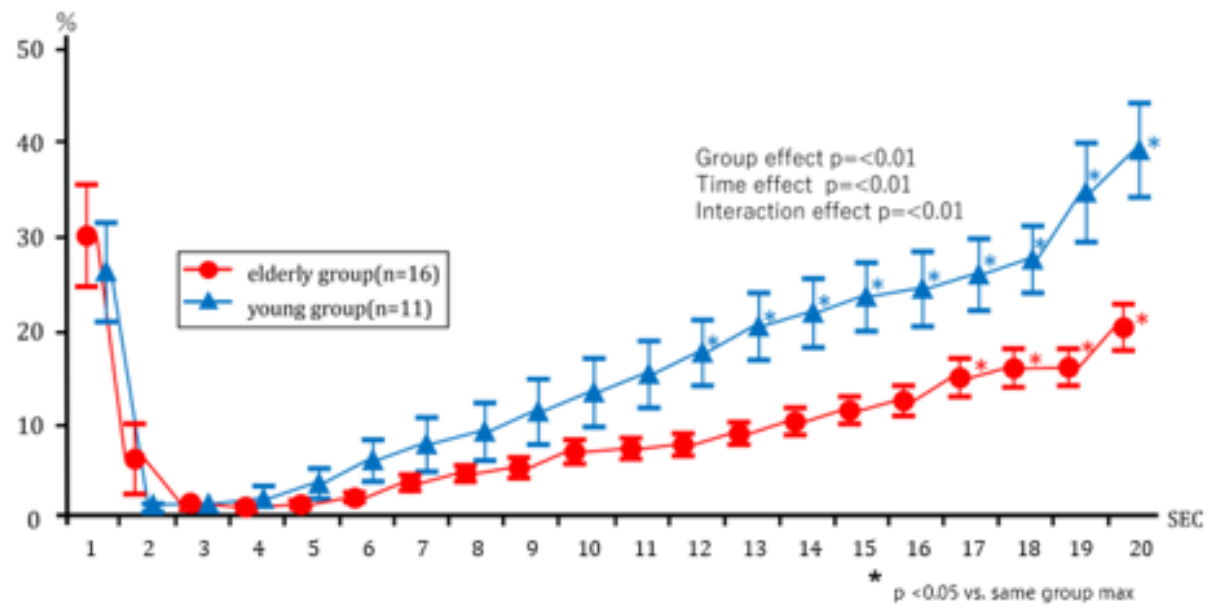

Figure 2: Rate of decrease per second relative to peak grip strength

\section{Discussion}

In this study, the hand grip endurance of the dominant hand in healthy elderly people was measured with maximum force for 20 seconds. Then, we examined the differences from young people, changes with time (seconds), changes in ED due to lifestyle, and grip strength measurement time.

\section{Handgrip endurance within elderly people}

It has been reported that endurance differs depending on the BMI range for young people[12], but in this experiment, 
no difference was observed between the elderly and young people depending on the BMI range, especially BMI 25 or higher (interaction effect $\mathrm{p}=0.95$ ).

Jakobsen et al. report that grip strength endurance is not a valid measure for assessing physical activity or quality of life in healthy subjects[13]. Miyahara et al. report that subjects performing household chores are more likely to maintain their physical activity and are less likely to lose their physical activity even with a state of emergency declared by COVID-19[14]. We calculated the difference in endurance of grip strength due to the engagement of housework in this case but found no difference in the decrease in endurance per second (interaction effect $\mathrm{p}=0.90$ ).

\section{Maximum grip strength measurement time}

Grip strength is said to have a significantly higher risk of total mortality, cardiovascular mortality, and other mortality as the aging decline increases[15,16]. In the reports on grip strength measurement, there are various reports that the grip strength measurement time is not set, and it is 3 to 5 seconds (17). The maximum muscle strength in this case was maximum 3 seconds after the start of measurement. From the above, it is desirable to measure about 5 seconds to measure the maximum muscle strength.

\section{Conclusion}

The elderly group did not show a significant decrease in the duration of grip strength for 20 seconds and exhibited a certain weak force. When it is required to exert momentary force, such as opening and closing the lid of a bottle in daily life, if this cannot be achieved, it may be necessary to take measures such as using selfhelp tools. In the future, we would like to find out whether there is a relationship between hand grip endurance and other physical function endpoints and cardiovascular risk (increased blood pressure).

\section{Acknowledgement}

None.

\section{Conflict of Interest}

None.

\section{References}

1. Chew STH, Kayambu G, Lew CCH, Ng TP, Ong F, et al. (2021) Singapore multidisciplinary consensus recommendations on muscle health in older adults: assessment and multimodal targeted intervention across the continuum of care. BMC Geriatr 21(1): 314.

2. Ellingsen O, Halle M, Prescott E, Linke A (2017) Response by Ellingsen et al to Letters Regarding Article, "High-Intensity Interval Training in Patients With Heart Failure With Reduced Ejection Fraction". Circulation 136(6): 611-612.
3. Hegde SM, Claggett B, Shah AM, Lewis EF, Anand I, et al. (2017) Physical Activity and Prognosis in the TOPCAT Trial (Treatment of Preserved Cardiac Function Heart Failure with an Aldosterone Antagonist). Circulation 136(11): 982-992.

4. Haupt M, Janner M, Richert F (2020) Impact of psychiatric disorders on the outcome of acute geriatric inpatient treatment: an exploratory investigation. Z Gerontol Geriatr 53(8): 749-755

5. Dent E, Lien C, Lim WS, Wong WC, Wong CH, et al. (2017) The AsiaPacific Clinical Practice Guidelines for the Management of Frailty. J Am Med Dir Assoc 18(7): 564-575.

6. Chen LK, Woo J, Assantachai P, Auyeung TW, Chou MY, et al. (2020) Asian Working Group for Sarcopenia: 2019 Consensus Update on Sarcopenia Diagnosis and Treatment. J Am Med Dir Assoc 21(3): 300-307.

7. McGrath R, Cawthon PM, Cesari M, Al Snih S, Clark BC (2020) Handgrip Strength Asymmetry and Weakness Are Associated with Lower Cognitive Function: A Panel Study. J Am Geriatr Soc 68(9): 2051-2058.

8. Inoue H, Watanabe H, Okami H, Shiraishi Y, Kimura A, et al. (2020) Handgrip strength correlates with walking in lumbar spinal stenosis. Eur Spine J 29(9): 2198-2204.

9. Larcher B, Zanolin-Purin D, Vonbank A, Heinzle CF, Mader A, et al. (2020) Usefulness of Handgrip Strength to Predict Mortality in Patients with Coronary Artery Disease. Am J Cardiol 129: 5-9.

10. Gerodimos V, Karatrantou K, Kakardaki K, Ioakimidis P (2021) Can maximal handgrip strength and endurance be improved by an 8-week specialized strength training program in older women? A randomized controlled study. Hand Surg Rehabil 40(2): 183-189.

11. Amaral JF, Mancini M, Novo Junior JM (2012) Comparison of three hand dynamometers in relation to the accuracy and precision of the measurements. Rev Bras Fisioter 16(3): 216-224.

12. Lad UP, Satyanarayana P, Shisode-Lad S, Siri ChC, Kumari NR (2013) A Study on the Correlation Between the Body Mass Index (BMI), the Body Fat Percentage, the Handgrip Strength and the Handgrip Endurance in Underweight, Normal Weight and Overweight Adolescents. J Clin Diagn Res 7(1): 51-54

13. Jakobsen LH, Rask IK, Kondrup J (2010) Validation of handgrip strength and endurance as a measure of physical function and quality of life in healthy subjects and patients. Nutrition 26(5): 542-550.

14. Miyahara S, Tanikawa Y, Hirai H, Togashi S (2021) Impact of the state of emergency enacted due to the COVID-19 pandemic on the physical activity of the elderly in Japan. J Phys Ther Sci 33(4): 345-350.

15. Gale CR, Martyn CN, Cooper C, Sayer AA (2007) Grip strength, body composition, and mortality. Int J Epidemiol 36(1): 228-235.

16. Rantanen T, Harris T, Leveille SG, Visser M, Foley D, et al. (2000) Muscle strength and body mass index as long-term predictors of mortality in initially healthy men. J Gerontol A Biol Sci Med Sci 55(3): M168-173.

17. New Physical Fitness Test Implementation Guidelines (2021) Ministry of Education, Culture, Sports, Science and Technology, Japan. 